\title{
Seroepidemiological and Microbiological Study of Brucellosis in Kuwait
}

\author{
Ts. Dimitrov ${ }^{a}$ D. Panigrahi ${ }^{b}$ M. Emara ${ }^{a}$ F. Awnia $\quad$ R. Passadilla ${ }^{a}$ \\ a Department of Laboratory Medicine (Microbiology Section), Infectious Diseases Hospital, b Department of \\ Medical Laboratory Sciences, Faculty of Allied Health Sciences, Kuwait University, Kuwait, Kuwait
}

\section{Key Words}

Brucellosis · Serological diagnosis · Seasonality • Antimicrobial susceptibility

\begin{abstract}
Objective: The primary objective of the study was to determine the prevalence of brucellosis and the antimicrobial susceptibility pattern of local Brucellae isolates in the Infectious Diseases Hospital, Kuwait. Subjects and Methods: A single serum sample was collected from each of 1,836 patients of different nationalities from January 2000 to December 2001. Any patient with a provisional diagnosis of fever or brucellosis had a standard tube agglutination (STA) test for the quantitation of Brucella antibodies. Blood cultures were done in 166 of 455 patients with significant STA titers, using the Bactec system. Antimicrobial susceptibility testing of 123 isolates of Brucella spp. was done against 8 antimicrobial agents. Results: A total of 455 serum samples (24.8\%) having an STA titer of $\geq 1: 160$ were presumptively diagnosed as cases of brucellosis. The peak isolation was in April and May. Brucella spp. were isolated from 123 blood cultures (74.1\%). The blood culture isolation rate was significantly higher in patients with an STA titer of $\geq 1: 1,280$ than in those with an STA titer of $\leq 1: 160(p<0.05)$. Antimicro-
\end{abstract}

bial susceptibility testing showed good in vitro activity of tetracycline, gentamicin, amikacin, streptomycin and ciprofloxacin against all isolates. Azithromycin had good anti-Brucella activity against only $42 \%$ of the isolates, while rifampicin and trimethoprim-sulfamethoxazole showed low in vitro anti-Brucella activity against 8 and $25 \%$ of the isolates, respectively. Conclusion: Brucellosis is quite common in Kuwait. Kuwaiti and Bangladeshi nationals were most affected. Significant titers on the STA test were detected in $24.8 \%$ of serum samples. Good in vitro activity against all isolates was found with tetracycline, gentamicin, amikacin, ciprofloxacin and streptomycin, and low activity with azithromycin, rifampicin and trimethoprim-sulfamethoxazole.

Copyright $@ 2004$ S. Karger AG, Basel

\section{Introduction}

Human brucellosis is an important zoonotic disease prevalent in all parts of the world including the Mediterranean basin, the Arabian Gulf and the Indian subcontinent $[1,2]$. In the last decade, brucellosis has changed dramatically from being an occupational illness to a foodborne disease. Since 1980, it has remained hyperendemic in Kuwait and has shown an increase in incidence since

\section{KARGER \\ Fax + 41613061234 \\ E-Mail karger@karger.ch \\ www. karger.com

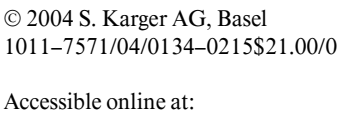

Dr. Ts. Dimitrov, MD

Specialist Microbiologist

Laboratory Department, Infectious Diseases Hospital

PO Box 4710, Safat 13048 (Kuwait)

Tel. +965 4809642, E-Mail dimitrov_varn90@hotmail.com 
$1990[3,4]$. The increase can be attributed mostly to an improvement in the diagnostic techniques and reporting of the disease [5]. Symptoms of human brucellosis are usually nonspecific; hence, laboratory tests are helpful for establishing the diagnosis. Though blood culture is the only specific test, its sensitivity ranges from 17 to $85 \%$, depending on the culture conditions and the bacterial strain. This method has good sensitivity for detecting $\mathrm{Bru}$ cella melitensis but low sensitivity for detecting Brucella abortus and Brucella suis [6]. For this reason, serological tests for diagnosing brucellosis have greater importance.

Despite the availability of many antibacterial agents, complete cure of the infection, without frequent relapses, is still an unattainable goal [7]. Brucella spp. are facultative intracellular parasites; therefore, complete eradication of the microorganism is difficult to achieve, and relapses are common. The best regime for the treatment of acute brucellosis is not definite. The fluoroquinolones and some of the new macrolides (azithromycin), because of their broad-spectrum antibacterial activity and effective intracellular penetration, could be candidates for the therapy of brucellosis [8-13]. In the present study, we report the incidence of brucellosis in Kuwait, the relationship of standard tube agglutination (STA) test titers to Brucella isolation from clinical samples and the antimicrobial susceptibility pattern of Brucellae isolates.

\section{Subjects and Methods}

The study was conducted from January 2000 to December 2001. During this period, a single serum sample was obtained from each of 1,836 patients with a history of pyrexia. Cases were provisionally diagnosed as 'brucellosis' where the patients presented with compatible clinical pictures like fever, night sweats, arthralgia and weight loss and also epidemiological evidence of exposure to a potential source of infection. Patients with fever but no symptoms compatible with brucellosis and no history of exposure were designated as 'fever for investigation'. The serum samples were tested by the STA for Brucella antibody titer using commercially available reagents (Plasmatec Lab Products, UK). A single blood sample was obtained for culture from 166 patients with high STA titers $(\geq 1: 160)$. The samples were injected into Bactec aerobic bottles (BD Bactec plus+Aero$\mathrm{bic} / \mathrm{F}$ ), and further incubation and detection of bacterial growth was done by an automated Bactec 9120 fluorescent series instrument system (Bactec Dickinson, USA), following the protocol of 21 days of incubation [14]. Isolates were identified as Brucella spp. in accordance with the taxonomic criteria of the International Committee of Nomenclature of Bacteria [15]. The sociodemographic characteristics of patients were obtained directly from the patients' records. Antimirobial susceptibility testing was performed by the standard disk diffusion method using brucella agar [16]. Commercially available antibiotic disks of tetracycline, streptomycin, gentamicin, amikacin, trimethroprim-sulfamethoxazole, rifampicin, ciprofloxacin and azithromycin were used. Since the disk diffusion method is not standardized for Brucellae, the results were not categorized as susceptible, intermediate or resistant. Instead, the diameter of the zone of inhibition was used to measure anti-Brucella activity. A zone size of $\leq 16 \mathrm{~mm}$ was considered to represent low activity, while that $>16 \mathrm{~mm}$ was considered to represent good activity.

For statistical evaluation of the results, Student's t test was used $(\mathrm{t}>1.96 ; \mathrm{p}<0.05)$.

\section{Results}

Of the 1,836 patients, 1,202 had fever for investigation, and of these, $110(9.2 \%)$ had a significant titer of $\geq 160$. 634 patients had brucellosis, and 345 of them $(54.4 \%)$ had an STA titer of $\geq 160$. Hence, of the 1,836 patients, $455(24.8 \%)$ had an STA titer of $\geq 160$. The distribution of the 455 seropositive patients according to nationality is shown in table 1 . Kuwaitis accounted for $68 \%$ and Bangladeshis for $16.5 \%$. The age of the patients varied between 10 and 80 years, with a mean age of 33 years. The peak months of isolation were April and May, accounting for $41.8 \%$ of the cases (fig. 1). Brucella spp. were isolated from 123 blood cultures (74.1\%). A statistically significant rate of Brucella isolation from blood was observed in patients having STA titers of $\geq 1: 1,280$ (49$39.8 \%$ ), in comparison to patients with STA titers of $\leq 1: 160(12-9.7 \% ; \mathrm{p}<0.05)$ (fig. 2$)$. Table 2 shows the results of antibacterial activity testing of the antimicrobials. Tetracycline, gentamicin, amikacin, streptomycin and ciprofloxacin had good anti-Brucella activity against all the isolates (zone of inhibition $>16 \mathrm{~mm}$ ). Azithromycin had good anti-Brucella activity against only $42 \%$ of the isolates. Rifampicin and trimethoprim-sulfamethoxazole showed variable in vitro anti-Brucella activity, and their potency was low against 8 and $25.2 \%$ of the isolates, respectively.

\section{Discussion}

Brucellosis has a worldwide distribution, including the Arabian peninsula $[1,2]$. It is one of the most commonly reported infectious diseases in Kuwait. During the 2-year period of this study, a total of 455 seropositive cases were detected. The disease was mostly prevalent among individuals in close contact with animals and persons having a history of consumption of raw milk and milk products, most probably accounting for the high incidence of brucellosis among Kuwaitis and Bangladeshis. In addition, Bangladeshi males are exclusively shepherds. In a pre- 
Fig. 1. Distribution of 455 STA-positive patients according to the time of serum collection.

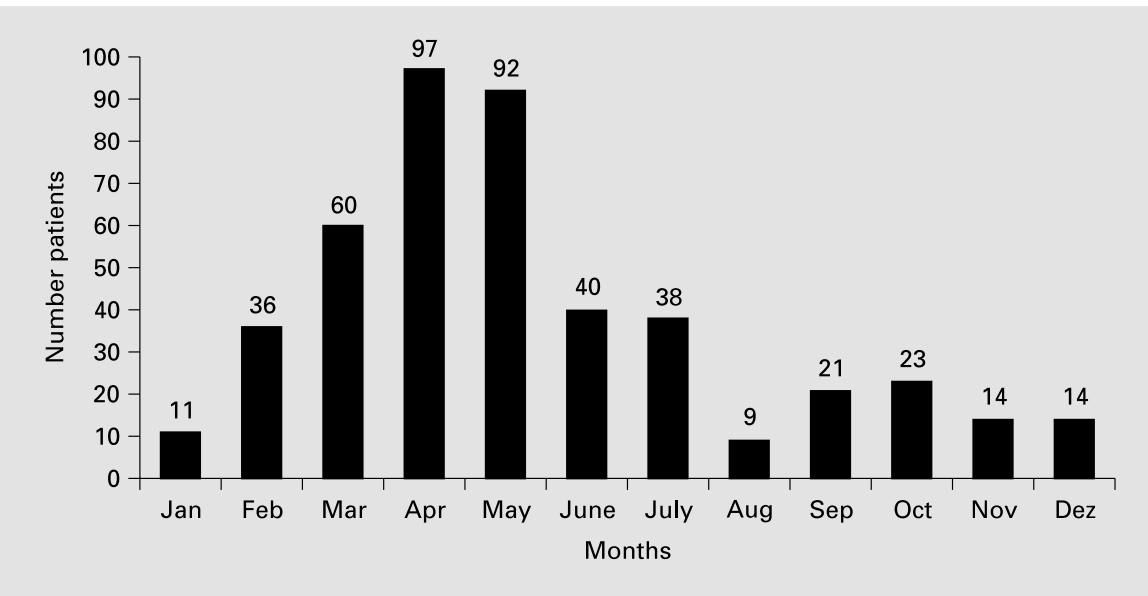

Table 1. Distribution of Brucella STA test titers according to nationality and clinical presentation

\begin{tabular}{|c|c|c|c|c|c|c|c|c|c|}
\hline \multirow[t]{2}{*}{ Nationality } & \multicolumn{3}{|c|}{ Fever for investigation alone } & \multicolumn{3}{|c|}{ Brucellosis alone } & \multirow{2}{*}{$\begin{array}{l}\text { Total number } \\
\text { of STA tests } \\
\text { (FFI + brucellosis) } \\
\mathrm{n}_{1}+\mathrm{n}_{3}\end{array}$} & \multicolumn{2}{|c|}{$\begin{array}{l}\text { Total number of STA tests } \\
\text { with titer of }>1: 160 \\
\text { (FFI + brucellosis) }\end{array}$} \\
\hline & $\mathrm{n}_{1}$ & $\mathrm{n}_{2}$ & $\%$ & $\mathrm{n}_{3}$ & $\mathrm{n}_{4}$ & $\%$ & & $\mathrm{n}_{2}+\mathrm{n}_{4}$ & $\%$ \\
\hline Kuwaitis & 390 & 55 & 14.1 & 468 & 255 & 54.5 & 858 & 310 & 36.0 \\
\hline Bangladeshi & 196 & 30 & 15.3 & 82 & 45 & 54.8 & 278 & 75 & 27.0 \\
\hline Indian & 280 & 8 & 2.8 & 7 & 2 & 28.6 & 287 & 10 & 3.0 \\
\hline OANGC & 131 & 3 & 2.3 & 19 & 12 & 63.2 & 150 & 15 & 10.0 \\
\hline OAGC & 44 & 9 & 20.5 & 47 & 27 & 57.4 & 91 & 36 & 39.0 \\
\hline Sri Lankan & 20 & 3 & 15.0 & 4 & 3 & 75.0 & 91 & 36 & 39.0 \\
\hline Pakistani & 80 & 1 & 1.2 & 1 & 0 & - & 87 & 1 & 1.1 \\
\hline Other nationalities & 55 & 1 & 1.8 & 6 & 1 & 16.6 & 61 & 2 & 3.3 \\
\hline Total & 1,202 & 110 & & 634 & & 345 & 1,836 & 455 & \\
\hline
\end{tabular}

FFI $=$ Fever for investigation; OANGC $=$ other Arab non-Gulf countries; OAGC $=$ other Arab Gulf countries.

$\mathrm{n}_{1}+\mathrm{n}_{3}=$ Total number of STA tests performed; $\mathrm{n}_{2}+\mathrm{n}_{4}=$ total number of STA tests with significant titer of $>1: 160$.

vious report from Kuwait in 1988 , the male to female ratio of brucellosis was reported to be $2: 1$ [3], but in the present study, the ratio was 10:1. The possible explanation for this change could be that, after the liberation of Kuwait in 1990, a lot more Bangladeshi male workers were employed as cattle rearers. This could have reduced the chances of Kuwaiti females taking care of the cattle. Brucellosis traditionally affects young to middle-aged individuals $[17,18]$. Our results confirm this, as two thirds of confirmed cases in this study were in the age group of 20-45 years.

In the last decade, brucellosis has changed dramatically from being an occupational disease to a food-borne ill- ness. Consumption of fresh and unpasteurized dairy products, especially cheese made from goat and sheep milk, are high risk factors for brucellosis in Saudi Arabia [19]. The case histories of the confirmed (positive blood culture) cases in this study showed that all these patients had a history of exposure to unpasteurized diary products.

Brucellosis is more frequent during late spring to early summer. During these months, the birth rates in cattle are very high, leading to increased environmental contamination and also availability of milk and milk products. A similar seasonal distribution of brucellosis occurred in the present study (fig. 1). 
Fig. 2. Recovery of Brucellae from blood in

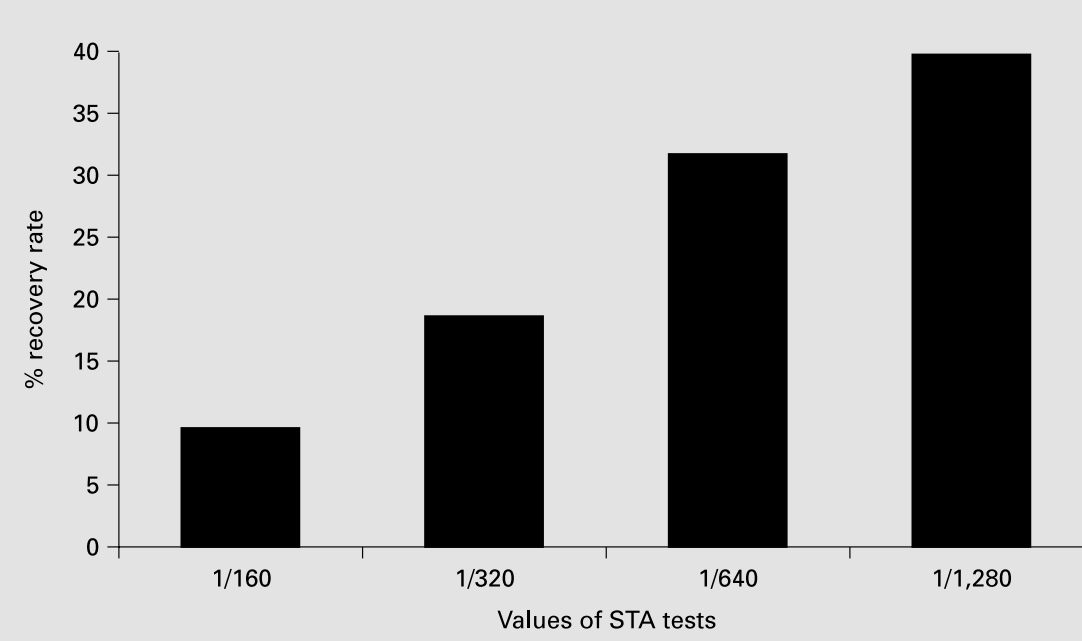
relation to STA titers.

Table 2. In vitro antimicrobial activity of 8 antimicrobial agents

\begin{tabular}{|c|c|c|c|c|c|c|c|c|}
\hline \multirow[t]{3}{*}{ Antibiotic } & \multicolumn{8}{|c|}{ Zone of inhibition } \\
\hline & \multicolumn{2}{|c|}{$<16 \mathrm{~mm}$} & \multicolumn{2}{|c|}{$17-20 \mathrm{~mm}$} & \multicolumn{2}{|c|}{$21-30 \mathrm{~mm}$} & \multicolumn{2}{|c|}{$>30 \mathrm{~mm}$} \\
\hline & $\mathrm{n}$ & $\%$ & $\mathrm{n}$ & $\%$ & $\mathrm{n}$ & $\%$ & $\mathrm{n}$ & $\%$ \\
\hline Tetracycline & 0 & - & 0 & - & 4 & 3.0 & 119 & 97.0 \\
\hline Gentamicin & 0 & - & 0 & - & 14 & 11.0 & 109 & 89.0 \\
\hline Amikacin & 0 & - & 0 & - & 14 & 11.0 & 109.0 & 89.0 \\
\hline Streptomycin & 0 & - & 0 & - & 58 & 47.0 & 65.0 & 53.0 \\
\hline Rifampicin & 10 & 8 & 68 & 55.3 & 45 & 36.0 & 0 & - \\
\hline Trimethoprim-sulfamethoxazole & 31 & 25.2 & 46 & 37.4 & 46 & 37.4 & 0 & - \\
\hline Ciprofloxacin & 0 & - & 0 & - & 116 & 94.3 & 7 & 5.7 \\
\hline Azithromycin & 71 & 57.7 & 35 & 28.5 & 14 & 11.4 & 3 & 2.4 \\
\hline
\end{tabular}

Figures show the number and percentage of strains.

The gold standard for diagnosis of brucellosis is isolation of the organism from blood, bone marrow or other tissues. The STA test is done as a screening test for presumptive diagnosis of brucellosis. Although the majority of brucellosis patients show an STA titer $\geq 1: 160$ and above, no single titer is always diagnostic. However, the agglutination test correlates well with culture positivity; the higher the STA titer, the more likely the isolation of Brucella spp. Accordingly, our study showed that the isolation rate from blood culture was significantly higher when the STA titer was $>1: 1,280$ than when the titer was $1: 160(\mathrm{p}<0.05)$.
There has been no unanimity on the most appropriate antibiotic therapy for human brucellosis. The evaluation of the efficacy of different antibiotic schedules is conditioned by the characteristics of the disease. Tetracyclines have remained the most effective antibiotics against brucellosis [7, 10, 20, 21]. Aminoglycosides penetrate human cells rather poorly, but have shown some intracellular activity after prolonged incubation. They have also been shown to have a substantial synergistic effect with tetracycline against Brucellae in in vitro and experimental studies and also in treatment of clinical brucellosis $[2,10]$. In the present study, tetracycline, gentamicin, amikacin and 
streptomycin showed good in vitro activity against all 123 strains of Brucellae. Rifampicin and trimethoprim-sulfamethoxazole have been suggested as an alternative regime, having satisfactory anti-Brucella activity in vitro as well as good tissue diffusion and the capacity to establish high intracellular concentrations [10, 16]. In this study, rifampicin and trimethoprim-sulfamethoxazole had good activity against 92 and $74.8 \%$ of the isolates, respectively. The potency of the two drugs was low against 8 and $25.2 \%$ of the strains, respectively. The fluoroquinolones possess excellent bactericidal activity against a variety of bacteria and also penetrate leukocytes and macrophages [22]. The encouraging results available from both experimental and clinical studies in relation to successful treatment of other intracellular infections, such as Legionella pneumophila, Chlamydia trachomatis and rickettsial infections, by fluoroquinolones and newer macrolides (azithromycin) called for their evaluation in the treatment of brucellosis. However, despite good in vitro activity and intracellular penetration, second-generation fluoroquinolones have shown poor results in experimental studies and therapy of human brucellosis [23], and our present in vitro study is consistent with these findings. This is probably due to the fact that these agents lack effective bactericidal activity at the acidic $\mathrm{pH}$ of phagolysosomes. The newer macrolides, e.g. azithromycin, have been recommended by Felek et al. [13] as an alternative choice in the treatment of human brucellosis. Our results do not agree with this suggestion. Azithromycin showed low in vitro antimicrobial activity against $58 \%$ of the isolates. On the basis of our data, we recommend the time-tested regimens for treatment of human brucellosis.

\section{Conclusion}

Brucellosis is an important infectious disease in $\mathrm{Ku}-$ wait, with a high incidence among Kuwaitis and Bangladeshis. The tube agglutination test was found to be a reliable screening test; the higher the STA titer, the higher the blood culture isolation rate. In the group of patients with STA titers of $\geq 1: 1,280$, the isolation rate of Brucellae was significantly higher. Good in vitro activity against all of the 123 isolates of Brucellae tested was shown with tetracycline, amikacin, gentamicin, streptomycin and ciprofloxacin.

\section{References}

1 Young EJ: An overview of human brucellosis. Clin Infect Dis 1995;21:283-290.

2 Ariza J: Brucellosis: An update. The perspective from the Mediterranean Basin. Rev Med Microbiol 1999;10:125-135.

3 Lulu AR, Araj GF, Khateeb MI, Mustafa MY, Yusev AR, Fenech FF: Human brucellosis in Kuwait: A prospective study of 400 cases. Q J Med 1988;66:39-54.

4 World Health Organization: Brucellosis: Kuwait. Wkly Epidemiol Rec 1986;8:2.

5 Ministry of Health: Infectious and Parasitic Diseases in Kuwait: Trends and Differentials. Kuwait, Department of Medical Records and Statistics, 1999.

6 Diaz R, Moriyon I: Laboratory techniques in the diagnosis of human brucellosis; in Young EJ, Corbel MJ (eds): Brucellosis: Clinical and Laboratory Aspects. Boca Raton, CRC, 1989 , pp 73-83.

7 Cisneros JM, Viciana P, Colmenero J: Multicenter prospective study of treatment of Brucella melitensis brucellosis with doxycycline for 6 weeks plus streptomycin for 2 weeks. Antimicrob Agents Chemother 1990;34:881-883.

8 Lang R, Rubinstein E: Quinolones for the treatment of brucellosis. J Antimicrob Chemother 1992;29:357-363.
9 Akova M, Uzun O, Akalin HE, Hayran M, Unal S, Gur D: Quinolones in treatment of human brucellosis: Comparative trial of ofloxacin-rifampin versus doxycycline-rifampin. Antimicrob Agents Chemother 1993;37:18311834.

10 Wendel HH: Modern chemotherapy for brucellosis in humans. Rev Infect Dis 1990;12:10601093.

11 Domingo S, Gastearena I: Comparative activity of azithromycin and doxycycline against Brucella sp. infection in mice. J Antimicrob Chemother 1995;36:674-656.

12 Lang R, Shasha B, Ifrach N, Tinman S, Rubinstein $\mathrm{E}$ : Therapeutic effects of roxithromycin and azithromycin in experimental murine brucellosis. Chemotherapy 1994;40:252-255.

13 Felek S, Demerdag K, Kalkan A, Akbulur A: Therapeutic effects of rifampin and erythromycin in experimental murine brucellosis. Clin Microbiol Infect 2000;6:111-114.

14 Bannatyne RM, Jackson MC, Memish Z: Rapid diagnosis of Brucella bacteremia by using the Bactec9240 system. J Clin Microbiol 1997;35: 2673-2674.

15 Jones LM, Wundt W: International Committee on Nomenclature of Bacteria. Subcommittee on the Taxonomy of Brucella. Minutes of Meeting. Int J Syst Bacteriol 1971;21:126128.
16 National Committee for Clinical Laboratory Standards: Performance Standards for Antimicrobial Disk Susceptibility Tests. Wayne, National Committee for Clinical Laboratory Standards, 1997.

17 Young EJ: Clinical manifestations of human brucellosis; in Young EJ, Corbel MJ (eds): Brucellosis: Clinical and Laboratory Aspects. Boca Raton, CRC, 1989, pp 97-126.

18 Taylor JP, Perdue JN: The changing epidemiology of human brucellosis in Texas, 19771986. Am J Epidemiol 1989;130:160-165.

19 Cooper CW: Risk factors in transmission of brucellosis from animals to humans in Saudi Arabia. Trans R Soc Trop Med Hyg 1992;86: 206-209.

20 Farrel ID, Hirshliffe PM, Robertson L: Sensitivity of Brucella spp. to tetracycline and its analogues. J Clin Pathol 1976;29:1097-1100.

21 Mortensen JE, Moore DG: Antimicrobial susceptibility of clinical isolates of Brucella. Diagn Microbiol Infect Dis 1986:5:163-169.

22 Easmon CS, Crane JP: Effect of ciprofloxacin in intracellular organisms: In-vitro and in-vivo studies. J Antimicrob Chemother 1986; 18(suppl D):43-48.

23 Garcia-Rodrigies JA, Carcia-Sanchez JE: Lack of effective bacterial activity of new quinolones against Brucella spp. Antimicrob Agents Chemother 1991;35:756-759. 